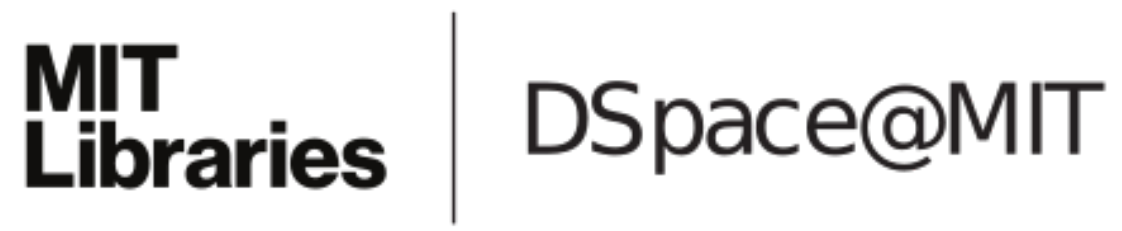

MIT Open Access Articles

The Pochoir Stencil Compiler

The MIT Faculty has made this article openly available. Please share how this access benefits you. Your story matters.

Citation: Tang, Yuan et al. "The Pochoir Stencil Compiler." SPAA '11 Proceedings of the 23rd ACM symposium on Parallelism in algorithms and architectures 2011. 117.

As Published: http://dx.doi.org/10.1145/1989493.1989508

Publisher: Association for Computing Machinery (ACM)

Persistent URL: http://hdl.handle.net/1721.1/72122

Version: Author's final manuscript: final author's manuscript post peer review, without publisher's formatting or copy editing

Terms of use: Creative Commons Attribution-Noncommercial-Share Alike 3.0 


\title{
The Pochoir Stencil Compiler
}

\author{
Yuan Tang Rezaul Chowdhury Bradley C. Kuszmaul \\ Chi-Keung Luk Charles E. Leiserson \\ MIT Computer Science and Artificial Intelligence Laboratory \\ Cambridge, MA 02139, USA
}

\begin{abstract}
A stencil computation repeatedly updates each point of a $d$ dimensional grid as a function of itself and its near neighbors. Parallel cache-efficient stencil algorithms based on "trapezoidal decompositions" are known, but most programmers find them difficult to write. The Pochoir stencil compiler allows a programmer to write a simple specification of a stencil in a domain-specific stencil language embedded in $\mathrm{C}++$ which the Pochoir compiler then translates into high-performing Cilk code that employs an efficient parallel cache-oblivious algorithm. Pochoir supports general $d$-dimensional stencils and handles both periodic and aperiodic boundary conditions in one unified algorithm. The Pochoir system provides a $\mathrm{C}++$ template library that allows the user's stencil specification to be executed directly in $\mathrm{C}++$ without the Pochoir compiler (albeit more slowly), which simplifies user debugging and greatly simplified the implementation of the Pochoir compiler itself. A host of stencil benchmarks run on a modern multicore machine demonstrates that Pochoir outperforms standard parallelloop implementations, typically running 2-10 times faster. The algorithm behind Pochoir improves on prior cache-efficient algorithms on multidimensional grids by making "hyperspace" cuts, which yield asymptotically more parallelism for the same cache efficiency.
\end{abstract}

\section{Categories and Subject Descriptors}

D.1.3 [Programming Techniques]: Concurrent ProgrammingParallel programming; D.3.2 [Programming Languages]: Language Classifications-Specialized application languages; G.4 [Mathematical Software]: Algorithm design and analysis.

This work was supported in part by a grant from Intel Corporation and in part by the National Science Foundation under Grants CCF-0937860 and CNS-1017058.

Yuan Tang is Assistant Professor of Computer Science at Fudan University in China and a Visiting Scientist at MIT CSAIL. Bradley C. Kuszmaul is Research Scientist at MIT CSAIL and Chief Architect at Tokutek, Inc. Chi-Keung Luk is Senior Staff Engineer at Intel Corporation and a Research Affiliate at MIT CSAIL. Rezaul Chowdhury is Research Scientist at Boston University and Research Affiliate at MIT CSAIL. Charles E. Leiserson is Professor of Computer Science and Engineering at MIT CSAIL.

Permission to make digital or hard copies of all or part of this work for personal or classroom use is granted without fee provided that copies are not made or distributed for profit or commercial advantage and that copies bear this notice and the full citation on the first page. To copy otherwise, to republish, to post on servers or to redistribute to lists, requires prior specific permission and/or a fee.

SPAA'11, June 4-6, 2011, San Jose, California, USA

Copyright 2011 ACM 978-1-4503-0743-7/11/06 ...\$10.00.

\section{General Terms}

Algorithms, Languages, Performance.

\section{Keywords}

$\mathrm{C}++$, cache-oblivious algorithm, Cilk, compiler, embedded domain-specific language, multicore, parallel computation, stencil, trapezoidal decomposition.

\section{INTRODUCTION}

Pochoir (pronounced "PO-shwar") is a compiler and runtime system for implementing stencil computations on multicore processors. A stencil defines the value of a grid point in a $d$-dimensional spatial grid at time $t$ as a function of neighboring grid points at recent times before $t$. A stencil computation $[2,9,11,12,16,17,26-$ $28,33,34,36,40,41]$ computes the stencil for each grid point over many time steps.

Stencil computations are conceptually simple to implement using nested loops, but looping implementations suffer from poor cache performance. Cache-oblivious $[15,38]$ divide-and-conquer stencil codes $[16,17]$ are much more efficient, but they are difficult to write, and when parallelism is factored into the mix, most application programmers do not have the programming skills or patience to produce efficient multithreaded codes.

As an example, consider how the 2D heat equation [13]

$$
\frac{\partial u_{t}(x, y)}{\partial t}=\alpha\left(\frac{\partial^{2} u_{t}(x, y)}{\partial x^{2}}+\frac{\partial^{2} u_{t}(x, y)}{\partial y^{2}}\right)
$$

on an $X \times Y$ grid, where $u_{t}(x, y)$ is the heat at a point $(x, y)$ at time $t$ and $\alpha$ is the thermal diffusivity, might be solved using a stencil computation. By discretizing space and time, this partial differential equation can be solved approximately by using the following Jacobi-style update equation:

$$
\begin{aligned}
u_{t+1}(x, y)= & u_{t}(x, y) \\
& +\frac{\alpha \Delta t}{\Delta x^{2}}\left(u_{t}(x-1, y)+u_{t}(x+1, y)-2 u_{t}(x, y)\right) \\
& +\frac{\alpha \Delta t}{\Delta y^{2}}\left(u_{t}(x, y-1)+u_{t}(x, y+1)-2 u_{t}(x, y)\right) .
\end{aligned}
$$

One simple parallel program to implement a stencil computation based on this update equation is with a triply nested loop, as shown in Figure 1 . The code is invoked as LoOPS $(u ; 0, T ; 0, X ; 0, Y)$ to perform the stencil computation over $T$ time steps. Although the loop indexing the time dimension is serial, the loops indexing the spatial dimensions can be parallelized, although as a practical matter, only the outer loop needs to be parallelized. There is generally no need to store the entire space-time grid, and so the code uses two 


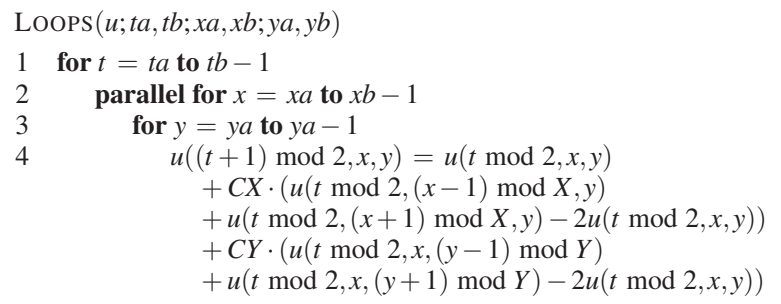

Figure 1: A parallel looping implementation of a stencil computation for the $2 \mathrm{D}$ heat equation with periodic boundary conditions. The array $u$ keeps two copies of an $X \times Y$ array of grid points, one for time $t$ and one for time $t+1$. The parameters $t a$ and $t b$ are the beginning and ending time steps, and $x a, x b, y a$, and $y b$ are the coordinates defining the region of the array $u$ on which to perform the stencil computation. The constants $C X=\alpha \Delta t / \Delta x^{2}$ and $C Y=\alpha \Delta t / \Delta y^{2}$ are precomputed. The call $\operatorname{LoOPS}(u ; 0, T ; 0, X ; 0, Y)$ performs the stencil computation over the whole $2 \mathrm{D}$ array for $T$ time steps.

copies of the spatial grid, swapping their roles on alternate time steps. This code assumes that the boundary conditions are periodic, meaning that the spatial grid wraps around to form a torus, and hence the index calculations for $x$ and $y$ are performed modulo $X$ and $Y$, respectively.

This loop nest is simple and fairly easy to understand, but its performance may suffer from poor cache locality. Let $\mathcal{M}$ be the number of grid points that fit in cache, and let $\mathcal{B}$ be the number of grid points that fit on a cache line. If the space grid does not fit in cache - that is, $X Y \gg \mathcal{M}$ - then this simple computation incurs $\Theta(T X Y / \mathcal{B})$ cache misses in the ideal-cache model [15].

Figure 2 shows the pseudocode for a more efficient cacheoblivious algorithm called TRAP, which is the basis of the algorithm used by the Pochoir compiler. We shall explain this algorithm in Section 3. It achieves $\Theta(T X Y / \mathcal{B} \sqrt{\mathcal{M}})$ cache misses, assuming that $X \approx Y$ and $T=\Omega(X)$. TRAP easily outperforms LOOPS on large data sets. For example, we ran both algorithms on a $5000 \times 5000$ spatial grid iterated for 5000 time steps using the Intel C++ version 12.0.0 compiler with Intel Cilk Plus [23] on a 12-core Intel Core 7 (Nehalem) machine with a private $32-\mathrm{KB}$ L1-data-cache, a private 256-KB L2-cache, and a shared 12-MB L3-cache. The code based on LoOPS ran in 248 seconds, whereas the Pochoir-generated code based on TRAP required about $24 \mathrm{sec}$ onds, more than a factor of 10 performance advantage.

Figure 3 shows Pochoir's performance on a wider range of benchmarks, including heat equation (Heat) [13] on a 2D grid, a 2D torus, and a 4D grid; Conway's game of Life (Life) [18]; 3D finite-difference wave equation (Wave) [32]; lattice Boltzmann method (LBM) [30]; RNA secondary structure prediction (RNA) $[1,6]$; pairwise sequence alignment (PSA) [19]; longest common subsequence (LCS) [7]; and American put stock option pricing (APOP) [24]. Pochoir achieves a substantial performance improvement over a straightforward loop parallelization for typical stencil applications, such as Heat and Life. Even LBM, which is a complex stencil having many states, achieves good speedup. When Pochoir does not achieve as much speedup over the loop code, it is often due to the spatial grid being too small to yield good parallelism, the innermost loop containing many branch conditionals, or the benchmark containing a high ratio of memory accesses to floating-point operations. For example, RNA's small grid size of $300^{2}$ yields a parallelism of just over 5 for both Pochoir and parallel loops, and its innermost loop contains many branch conditionals. PSA operates over a diamond-shaped domain, and so the application employs many conditional branches in the kernel in order to distinguish interior points from exterior points. These overheads

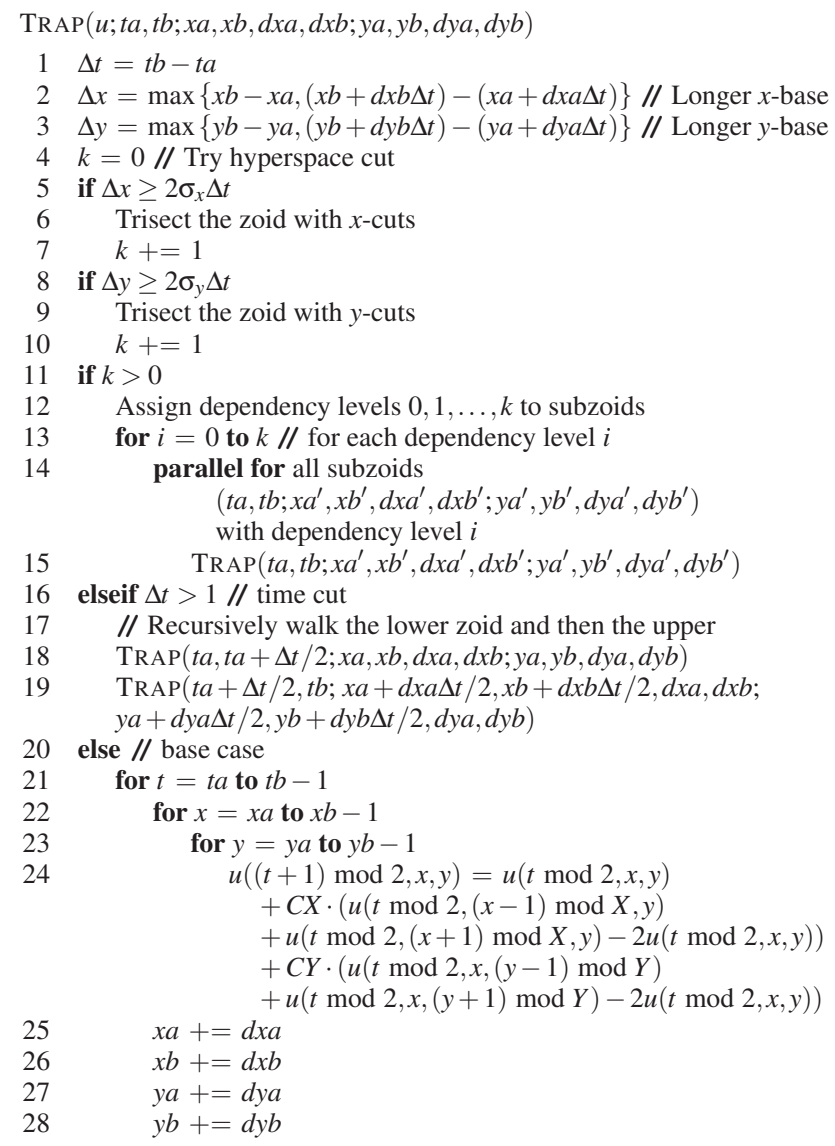

Figure 2: The Pochoir cache-oblivious algorithm that implements a $2 \mathrm{D}$ stencil computation to solve the $2 \mathrm{D}$ heat equation using a trapezoidal decomposition with hyperspace cuts. The parameter $u$ is an $X \times Y$ array of grid points. The remaining variables describe the hypertrapezoid, or "zoid," embedded in space-time that is being processed: $t a$ and $t b$ are the beginning and ending time steps; $x a, x b, y a$, and $y b$ are the coordinates defining the base of the zoid; $d x a, d x b, d y a$, and $d y b$ are the slopes (actually inverse slopes) of the sides of the zoid. The values $\sigma_{x}$ and $\sigma_{y}$ are the slopes of the stencil in the $x$ - and $y$-dimensions, respectively, which are both 1 for the heat equation.

can sometimes significantly mitigate a cache-efficient algorithm's advantage in incurring fewer cache misses.

The Berkeley autotuner $[8,26,41]$ focuses on optimizing the performance of stencil kernels by automatically selecting tuning parameters. Their work serves as a good benchmark for the maximum possible speedup one can get on a stencil. K. Datta and S. Williams graciously gave us their code for computing a 7-point stencil and a 27 -point stencil on a $258^{3}$ grid with "ghost cells" (see Section 4 ) using their system. Unfortunately, we were unable to reproduce the reported results from [8] — presumably because there were too many differences in hardware, compilers, and operating system and thus we are unable to offer a direct side-by-side comparison. Instead, we present in Figure 5 a comparison of our results to their reported results.

We tried to make the operating conditions of the Pochoir tests as similar as possible to the Berkeley environment reported in [8]. We compared Pochoir running 8 worker threads on a 12-core system to the reported numbers for the Berkeley autotuner running 8 threads on 8 cores. The comparison may result in a disadvantage to the Berkeley autotuner, because their reported numbers involve 


\begin{tabular}{|c|c|c|c|c|c|c|c|c|c|c|}
\hline \multirow[t]{2}{*}{ Benchmark } & \multirow[t]{2}{*}{ Dims } & \multirow{2}{*}{$\begin{array}{c}\text { Grid } \\
\text { size }\end{array}$} & \multirow{2}{*}{$\begin{array}{l}\text { Time } \\
\text { steps }\end{array}$} & \multicolumn{3}{|c|}{ Pochoir } & \multicolumn{2}{|c|}{ Serial loops } & \multicolumn{2}{|c|}{ 12-core loops } \\
\hline & & & & 1 core & 12 cores & speedup & time & ratio & time & ratio \\
\hline Heat & 2 & $16,000^{2}$ & 500 & $277 \mathrm{~s}$ & $24 s$ & 11.5 & $612 \mathrm{~s}$ & 25.5 & $149 \mathrm{~s}$ & 6.2 \\
\hline Heat & $2 p$ & $16,000^{2}$ & 500 & $281 \mathrm{~s}$ & $24 \mathrm{~s}$ & 11.7 & $1,647 \mathrm{~s}$ & 68.6 & $248 \mathrm{~s}$ & 10.3 \\
\hline Heat & 4 & $150^{4}$ & 100 & $154 \mathrm{~s}$ & $54 \mathrm{~s}$ & 2.9 & $433 \mathrm{~s}$ & 8.0 & $104 \mathrm{~s}$ & 1.9 \\
\hline Life & $2 p$ & $16,000^{2}$ & 500 & $345 \mathrm{~s}$ & $28 s$ & 12.3 & $2,419 \mathrm{~s}$ & 86.4 & $332 s$ & 11.9 \\
\hline Wave & 3 & $1,000^{3}$ & 500 & $3,082 \mathrm{~s}$ & $447 \mathrm{~s}$ & 6.9 & $3,170 \mathrm{~s}$ & 7.1 & $1,071 \mathrm{~s}$ & 2.4 \\
\hline LBM & 3 & $100^{2} \times 130$ & 3,000 & $345 \mathrm{~s}$ & $68 \mathrm{~s}$ & 5.1 & $304 \mathrm{~s}$ & 4.5 & $220 \mathrm{~s}$ & 3.2 \\
\hline RNA & 2 & $300^{2}$ & 900 & $90 \mathrm{~s}$ & $20 \mathrm{~s}$ & 4.5 & $121 \mathrm{~s}$ & 6.1 & $26 \mathrm{~s}$ & 1.3 \\
\hline PSA & 1 & 100,000 & 200,000 & $105 \mathrm{~s}$ & $18 \mathrm{~s}$ & 5.8 & $432 s$ & 24.0 & $77 \mathrm{~s}$ & 4.3 \\
\hline LCS & 1 & 100,000 & 200,000 & $57 \mathrm{~s}$ & $9 \mathrm{~s}$ & 6.3 & $105 \mathrm{~s}$ & 11.7 & $27 \mathrm{~s}$ & 3.0 \\
\hline APOP & 1 & $2,000,000$ & 10,000 & $43 \mathrm{~s}$ & $4 s$ & 10.7 & $515 \mathrm{~s}$ & 128.8 & $48 \mathrm{~s}$ & 12.0 \\
\hline
\end{tabular}

Figure 3: Pochoir performance on an Intel Core i7 (Nehalem) machine. The stencils are nonperiodic unless the Dims column contains a "p." The header Serial loops means a serial for loop implementation running on one core, whereas 12-core loops means a parallel cilk_for loop implementation running on 12 cores. The header ratio indicates how much slower the looping implementation is than the 12-core Pochoir implementation. For nonperiodic stencils, the looping implementations employ ghost cells [8] to avoid boundary processing.

only a single time step, whereas the Pochoir code runs for 200 time steps. (It does not make sense to run Pochoir for only 1 time step, since its efficiency is in large measure due to the temporal locality of cache use.) Likewise, the Pochoir figures may exhibit a disadvantage compared with the Berkeley ones, because Pochoir had to cope with load imbalances due to the scheduling of 8 threads on 12 cores. Notwithstanding these issues, as can be seen from the figure, Pochoir's performance is generally comparable to that of the Berkeley autotuner on these two benchmarks.

The Pochoir-generated TRAP code is a cache-oblivious [15,38] divide-and-conquer algorithm based on the notion of trapezoidal decompositions introduced by Frigo and Strumpen [16, 17]. We improve on their code by using hyperspace cuts, which produce an asymptotic improvement in parallelism while attaining essentially the same cache efficiency. As can be seen from Figure 2, however, this divide-and-conquer parallel code is far more complex than LOOPS, involving recursion over irregular geometric regions. Moreover, TRAP presents many opportunities for optimization, including coarsening the base case of the recursion and handling boundary conditions. We contend that one cannot expect average application programmers to be able to write such complex high-performing code for each stencil computation they wish to perform.

The Pochoir stencil compiler allows programmers to write simple functional specification for arbitrary $d$-dimensional stencils, and then it automatically produces a highly optimized, cacheefficient, parallel implementation. The Pochoir language can be viewed as a domain-specific language $[10,21,31]$ embedded in the base language $\mathrm{C}++$ with the Cilk multithreading extensions [23].

As shown in Figure 4, the Pochoir system operates in two phases, only the second of which involves the Pochoir compiler itself. For the first phase, the programmer compiles the source program with the ordinary Intel $\mathrm{C}++$ compiler using the Pochoir template library, which implements Pochoir's linguistic constructs using unoptimized but functionally correct algorithms. This phase ensures that the source program is Pochoir-compliant. For the second phase, the programmer runs the source through the Pochoir compiler, which acts as a preprocessor to the Intel $\mathrm{C}++$ compiler, performing a source-to-source translation into a postsource $\mathrm{C}++$ program that employs the Cilk extensions. The postsource is then compiled with the Intel compiler to produce the optimized binary executable. The Pochoir compiler makes the following promise:

The Pochoir Guarantee: If the stencil program compiles and runs with the Pochoir template library during Phase 1 , no errors will occur during Phase 2 when it

\begin{tabular}{|c|c|c|}
\hline & Berkeley & Pochoir \\
\hline CPU & Xeon X5550 & Xeon X5650 \\
Clock & $2.66 \mathrm{GHz}$ & $2.66 \mathrm{GHz}$ \\
cores/socket & 4 & 6 \\
Total \# cores & 8 & 12 \\
Hyperthreading & Enabled & Disabled \\
L1 data cache/core & $32 \mathrm{~KB}$ & $32 \mathrm{~KB}$ \\
L2 cache/core & $256 \mathrm{~KB}$ & $256 \mathrm{~KB}$ \\
L3 cache/socket & $8 \mathrm{MB}$ & $12 \mathrm{MB}$ \\
Peak computation & $85 \mathrm{GFLOPS}$ & $120 \mathrm{GFLOPS}$ \\
\hline Compiler & icc 10.0 .0 & icc 12.0 .0 \\
Linux kernel & & 2.6 .32 \\
Threading model & Pthreads & Cilk Plus \\
\hline 3D 7-point & $2.0 \mathrm{GStencil} / \mathrm{s}$ & $2.49 \mathrm{GSStencil} / \mathrm{s}$ \\
8 cores & $15.8 \mathrm{GFLOPS}$ & $19.92 \mathrm{GFLOPS}$ \\
\hline 3D 27-point & $0.95 \mathrm{GStencil} / \mathrm{s}$ & $0.88 \mathrm{GStencil} / \mathrm{s}$ \\
8 cores & $28.5 \mathrm{GFLOPS}$ & $26.4 \mathrm{GFLOPS}$ \\
\hline
\end{tabular}

Figure 5: A comparison of Pochoir to the reported results from [8]. The 7-point stencil requires 8 floating-point operations per grid point, whereas the 27-point stencil requires 30 floating-point operations per grid point.

is compiled with the Pochoir compiler or during the subsequent running of the optimized binary.

Pochoir's novel two-phase compilation strategy allowed us to build significant domain-specific optimizations into the Pochoir compiler without taking on the massive job of parsing and typechecking the full $\mathrm{C}++$ language. Knowing that the source program compiles error-free with the Pochoir template library during Phase 1 allows the Pochoir compiler in Phase 2 to treat portions of the source as uninterpreted text, confident that the Intel compiler will compile it correctly in the optimized postsource. Moreover, the Pochoir template library allows the programmer to debug his or her code using a comfortable native $\mathrm{C}++$ tool chain without the complications of the Pochoir compiler.

Figure 6 shows the Pochoir source code for the periodic 2D heat equation. We leave the specification of the Pochoir language to Section 2, but outline the salient features of the language using this code as an example.

Line 7 declares the Pochoir shape of the stencil, and line 8 creates the 2-dimensional Pochoir object heat having that shape. The Pochoir object will contain all the state necessary to perform the computation. Each triple in the array 2D_five_pt corresponds to a relative offset from the space-time grid point $(t, x, y)$ that the stencil kernel (declared in lines 12-14) will access. The compiler cannot infer the stencil shape from the kernel, because the kernel can be arbitrary code, and accesses to the grid points can be hidden in subroutines. The Pochoir template library complains during Phase 1, however, if an access to a grid point during the kernel computation falls outside the region specified by the shape declaration. 


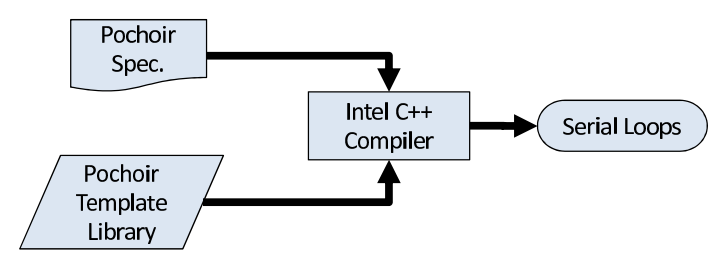

(a)

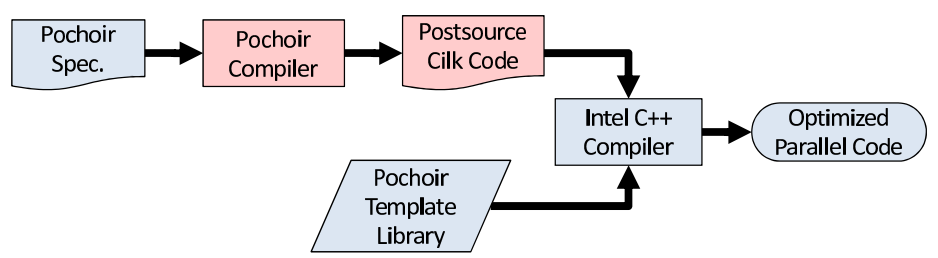

(b)

Figure 4: Pochoir's two-phase compilation strategy. (a) During Phase 1 the programmer uses the normal Intel C++ compiler to compile his or her code with the Pochoir template library. Phase 1 verifies that the programmer's stencil specification is Pochoir compliant. (b) During Phase 2 the programmer uses the Pochoir compiler, which acts as a preprocessor to the Intel C++ compiler, to generate optimized multithreaded Cilk code.

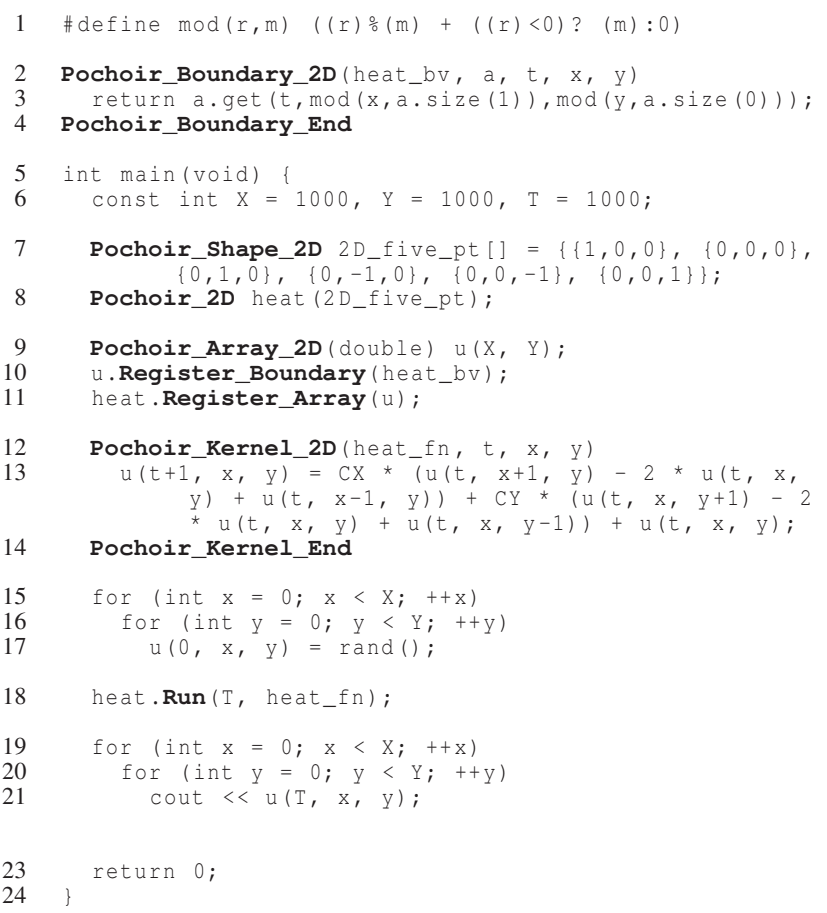

Figure 6: The Pochoir stencil source code for a periodic 2D heat equation. Pochoir keywords are boldfaced.

Line 9 declares $u$ as an $X \times Y$ Pochoir array of double-precision floating-point numbers representing the spatial grid. Lines $2-4$ define a boundary function that will be called when the kernel function accesses grid points outside the computing domain, that is, if it tries to access $u(t, x, y)$ with $x<0, x \geq X, y<0$, or $y \geq y$. The boundary function for this periodic stencil performs calculations modulo the dimensions of the spatial grid. (Section 2 shows how nonperiodic stencils can be specified, including how to specify Dirichlet and Neumann boundary conditions [14].) Line 10 associates the boundary function heat_bv with the Pochoir array u. Each Pochoir array has exactly one boundary function to supply a value when the computation accesses grid points outside of the computing domain. Line 11 registers the Pochoir array $u$ with the heat Pochoir object. A Pochoir array can be registered with more than one Pochoir object, and a Pochoir object can have multiple Pochoir arrays registered.

Lines 12-14 define a kernel function heat_fn, which specifies how the stencil is computed for every grid point. This kernel can be an arbitrary piece of code, but accesses to the registered Pochoir arrays must respect the declared shape(s).

Lines 15-17 initialize the Pochoir array $u$ with values for time step 0 . If a stencil depends on more than one prior step as indicated by the Pochoir shape, multiple time steps may need to be initialized.
Line 18 executes the stencil object heat for $T$ time steps using kernel function heat_fn. Lines 19-21 prints the result of the computation by reading the elements $u(T, x, y)$ of the Pochoir array. In fact, Pochoir overloads the " $<<$ " operator so that the Pochoir array can be pretty-printed by simply writing "cout $<$ u;".

The remainder of this paper is organized as follows. Section 2 provides a full specification of the Pochoir embedded language. Section 3 describes the cache-oblivious parallel algorithm used by the compiled code and analyzes its theoretical performance. Section 4 describes four important optimizations employed by the Pochoir compiler. Section 5 describes related work, and Section 6 offers some concluding remarks.

\section{THE POCHOIR SPECIFICATION LANGUAGE}

This section describes the formal syntax and semantics of the Pochoir language, which was designed with a view to offer as much expressiveness as possible without violating the Pochoir Guarantee. Since we wanted to allow third-party developers to implement their own stencil compilers that could use the Pochoir specification language, we avoided to the extent possible making the language too specific to the Pochoir compiler, the Intel C++ compiler, and the multicore machines we used for benchmarking.

The static information about a Pochoir stencil computation, such as the computing kernel, the boundary conditions, and the stencil shape, is stored in a Pochoir object, which is declared as follows:

- Pochoir_dimD name (shape);

This statement declares name as a Pochoir object with dim spatial dimensions and computing shape shape, where dim is a small positive integer and shape is an array of arrays which describes the shape of the stencil as elaborated below.

We now itemize the remaining Pochoir constructs and explain the semantics of each.

- Pochoir_Shape_dimD name [] $=\{$ cells $\}$

This statement declares name as a Pochoir shape that can hold shape information for dim spatial dimensions. The Pochoir shape is equivalent to an array of arrays, each of which contains $\operatorname{dim}+1$ integer numbers. These numbers represent the offset of each memory footprint in the stencil kernel relative to the space-time grid point $\langle t, x, y, \cdots\rangle$. For example, suppose that the computing kernel employs the following update equation:

$$
\begin{aligned}
u_{t}(x, y)= & u_{t-1}(x, y) \\
& +\frac{\alpha \Delta t}{\Delta x^{2}}\left(u_{t-1}(x-1, y)+u_{t-1}(x+1, y)-2 u_{t-1}(x, y)\right) \\
& +\frac{\alpha \Delta t}{\Delta y^{2}}\left(u_{t-1}(x, y-1)+u_{t-1}(x, y+1)-2 u_{t-1}(x, y)\right) .
\end{aligned}
$$

The shape of this stencil is $\{\{0,0,0\},\{-1,1,0\},\{-1,0,0\}$, $\{-1,-1,0\},\{-1,0,1\},\{-1,0,-1\}\}$. 
The first cell in the shape is the home cell, whose spatial coordinates must all be 0 . During the computation, this cell corresponds to the grid point being updated. The remaining cells must have time offsets that are smaller than the time coordinate of the home cell, and the corresponding grid points during the computation are read-only.

The depth of a shape is the time coordinate of the home cell minus the minimum time coordinate of any cell in the shape. The depth corresponds to the number of time steps on which a grid point depends. For our example stencil, the depth of the shape is 1 , since a point at time $t$ depends on points at time $t-1$.. If a stencil shape has depth $k$, the programmer must initialize all Pochoir arrays for time steps $0,1, \ldots, k-1$ before running the computation.

- Pochoir_Array_dim (type, depth) name $\left(\right.$ size $_{\text {dim-1 }}, \ldots$, size $_{1}$, size $_{0}$ )

This statement declares name as a Pochoir array of type type with dim spatial dimensions and a temporal dimension. The size of the $i$ th spatial dimension, where $i \in\{0,1, \ldots, \operatorname{dim}\}$, is given by $\operatorname{size}_{i}$. The temporal dimension has size $k+1$, where $k$ is the depth of the Pochoir shape, and are reused modulo $k+1$ as the computation proceeds. The user may not obtain an alias to the Pochoir array or its elements.

\section{- Pochoir_Boundary_dimD(name, array, idx $x_{t}, i d x_{d i m-1}$, $\left.\ldots, i d x_{1}, i d x_{0}\right)$ $\langle$ definition \\ Pochoir_Boundary_End}

This construct defines a boundary function called name that will be invokeda to supply a value when the stencil computation accesses a point outside the domain of the Pochoir array array. The Pochoir array array has dim spatial dimensions, and $\left\langle i d x_{d i m-1}, \ldots, i d x_{1}, i d x_{0}\right\rangle$ are the spatial coordinates of the given point outside the domain of array. The coordinate in the time dimension is given by $i d x_{t}$. The function body $\langle$ definition $\rangle$ ) is $\mathrm{C}++$ code that defines the values of array on its boundary. A current restriction is that this construct must be declared outside of any function, that is, the boundary function is declared global.

\section{- Pochoir_Kernel_dim $\mathbf{D}\left(\right.$ name, $\operatorname{array}, i d x_{t}, i d x_{d i m-1}, \ldots$, $\left.i d x_{1}, i d x_{0}\right)$ $\langle$ definition $\rangle$ \\ Pochoir_Kernel_End}

This construct defines a kernel function named name for updating a stencil on a spatial grid with dim spatial dimensions. The spatial coordinates of the point to update are $\left\langle i d x_{d i m-1}, \ldots, i d x_{1}, i d x_{0}\right\rangle$, and $i d x_{t}$ is the coordinate in time dimension. The function body $\langle$ definition $\rangle$ may contain arbitrary $\mathrm{C}++$ code to compute the stencil. Unlike boundary functions, this construct can be defined in any context.

\section{- name.Register_Array(array)}

A call to this member function of a Pochoir object name informs name that the Pochoir array array will participate in its stencil computation.

\section{- name.Register_Boundary (bdry)}

A call to this member function of a Pochoir array name associates the declared boundary function bdry with name. The boundary function is invoked to supply a value whenever an off-domain memory access occurs. Each Pochoir array is associated with exactly one boundary function at any given time, but the programmer can change boundary functions by registering a new one.

- name.Run $(T$, kern $)$
This function call runs the stencil computation on the Pochoir object name for $T$ time steps using computing kernel function kern.

After running the computation for $T$ steps, the results of the computation can be accessed by indexing its Pochoir arrays at time $T+k-1$, where $k$ is the depth of the stencil shape. The programmer may resume the running of the stencil after examining the result of the computation by calling name. $\operatorname{Run}\left(T^{\prime}\right.$, kern $)$, where $T^{\prime}$ is the number of additional steps to execute. The result of the computation is then in the computation's Pochoir arrays indexed by time $T+T^{\prime}+k-1$.

\section{Rationale}

The Pochoir language is a product of many design decisions, some of which were influenced by the current capabilities of the Intel 12.0.0 C++ compiler. We now discuss some of the more important design decisions.

Although we chose to pass a kernel function to the Run method of a Pochoir object, we would have preferred to simply store the kernel function with the Pochoir object. The kernel function is a C++ lambda function [5], however, whose type is not available to us. Thus, although we can pass the lambda function as a template type, we cannot store it unless we create a std: : function to capture its type. Since the Intel compiler does not yet support std: : function, this avenue was lost to us. There is only one kernel function per Pochoir object, however, and so we decided as a second-best alternative that it would be most convenient for users if they could declare a kernel function in any context and we just pass it as an argument to the Run member function.

The lack of support for function objects also had an impact on the declaration of boundary functions. We wanted to store each boundary function with a Pochoir array so that whenever an access to the array falls outside the computing domain, we can call the boundary function to supply a value. The only way to create a function that can be stored is to use an ordinary function, which must be declared in a global scope. We hope to improve Pochoir's linguistic design when function objects are fully supported by the compiler.

We chose to specify the kernel function imperatively rather than as a pure function or as an expression that returns a value for the grid point being updated. This approach allows a user to write multiple statements in a kernel function and provides flexibility on how to specify a stencil formula. For example, the user can choose to specify a stencil formula as $a(t, i, j)=\ldots$ or $a(t+1, i, j)=\ldots$, whichever is more convenient.

We chose to make the user copy data in and out of Pochoir internal data structures, rather than operate directly on the user's arrays. Since the user is typically running the stencil computation for many time steps, we decided that the copy-in/copy-out approach would not cause much overhead. Moreover, the layout of data is now under the control of the compiler, allowing it to optimize the storage for cache efficiency.

\section{POCHOIR'S CACHE-OBLIVIOUS PARALLEL ALGORITHM}

This section describes the parallel algorithm at the core of Pochoir's efficiency. TRAP is a cache-oblivious algorithm based on "trapezoidal decompositions" [16, 17], but which employs a novel "hyperspace-cut" strategy to improve parallelism without sacrificing cache-efficiency. On a $d$-dimensional spatial grid with all "normalized" spatial dimensions equal to $w$ and the time dimension a power-of-2 multiple of $w$, TRAP achieves $\Theta\left(w^{d-\lg (d+2)+1} / d^{2}\right)$ parallelism, whereas Frigo and Strumpen's 
original parallel trapezoidal decomposition algorithm [17] achieves $\Theta\left(w^{d-\lg \left(2^{d}+1\right)+1} / 2^{d}\right)=O(w)$ parallelism. Both algorithms exhibit the same asymptotic cache complexity of $\Theta\left(h w^{d} / \mathcal{M}^{1 / d} \mathcal{B}\right)$ proved by Frigo and Strumpen, where $h$ is the height of the time dimension, $\mathcal{M}$ is the cache size, and $\mathcal{B}$ is the cache-block size.

TRAP uses a cache-oblivious [15] divide-and-conquer strategy based on a recursive trapezoidal decomposition of the space-time grid, which was introduced by Frigo and Strumpen [16]. They originally used the technique for serial stencil computations, but later extended it to parallel stencil computations [17]. Whereas Frigo and Strumpen's parallel algorithm cuts the spatial dimensions of a hypertrapezoid, or "zoid," one at a time with "parallel space cuts," TRAP performs a hyperspace cut where it applies parallel space cuts simultaneously to as many dimensions as possible, yielding asymptotically more parallelism when the number of spatial dimensions is 2 or greater. As we will argue later in this section, TRAP achieves this improvement in parallelism while attaining the same cache complexity as Frigo and Strumpen's original parallel algorithm.

TRAP operates as follows. Line 5 of Figure 2 determines whether the $x$-dimension of the zoid can be cut with a parallel space cut, and if so, line 6 trisects the zoid, as we shall describe later in this section and in Figure 7, but it does not immediately spawn recursive tasks to process the subzoids, as Frigo and Strumpen's algorithm would. Instead, the code attempts to make a "hyperspace cut" by proceeding to the $y$-dimension, and if there were more dimensions, to those, cutting as many dimensions as possible before spawning recursive tasks to handle the subzoids. The counter $k$ keeps track of how many spatial dimensions are cut. If $k>0$ spatial dimensions are trisected, as tested for in line 11, then line 12 assigns each subzoid to one of $k+1$ dependency levels such that the subzoids assigned to the same level are independent and can be processed in parallel, as we describe later in this section and in Figure 8. Lines 13-15 recursively walk all subzoids level by level in parallel. Lines 17-19 perform a time cut if no space cut can be performed. Lines 20-28 perform the base-case computation if the zoid is sufficiently small that no space or time cut is productive.

We first introduce some notations and definitions, many of which have been borrowed or adapted from [16, 17]. A $(d+1)$-dimensional space-time hypertrapezoid, or $(d+1)$ zoid, $Z=\left(t a, t b ; x a_{0}, x b_{0}, d x a_{0}, d x b_{0} ; x a_{1}, x b_{1}, d x a_{1}, d x b_{1} ; \ldots\right.$; $\left.x a_{d-1}, x b_{d-1}, d x a_{d-1}, d x b_{d-1}\right)$, where all variables are integers, is the set of integer grid points $\left\langle t, x_{0}, x_{1}, \ldots, x_{d-1}\right\rangle$ such that $t a \leq$ $t<t b$ and $x a_{i}+d x a_{i}(t-t a) \leq x_{i}<x b_{i}+d x b_{i}(t-t a)$ for all $i \in\{0,1, \ldots, d-1\}$. The height of $Z$ is $\Delta t=t b-t a$. Define the projection trapezoid $Z_{i}$ of $Z$ along spatial dimension $i$ to be the $2 \mathrm{D}$ trapezoid that results from projecting the zoid $z$ onto the dimensions $x_{i}$ and $t$. The projection trapezoid $z_{i}$ has two bases (sides parallel to the $x_{i}$ axis) of lengths $\Delta x_{i}=x b_{i}-x a_{i}$ and $\nabla x_{i}=\left(x b_{i}+d x b_{i} \Delta t\right)-\left(x a_{i}+d x a_{i} \Delta t\right)$. We define the $\boldsymbol{w i d t h}^{1} w_{i}$ of $z_{i}$ to be the length of the longer of the two bases (parallel sides) of $Z_{i}$, that is $w_{i}=\max \left\{\Delta x_{i}, \nabla x_{i}\right\}$. The value $w_{i}$ is also called the width of $Z$ along spatial dimension $i$. We say that $Z_{i}$ is upright if $w_{i}=\Delta x_{i}$ - the longer base corresponds to time $t a-$ and inverted otherwise. A zoid $Z$ is well-defined if its height is positive, its widths along all spatial dimensions are positive, and the lengths of its bases along all spatial dimensions are nonnegative. A projection trapezoid $Z_{i}$ is minimal if $Z_{i}$ is upright and $\nabla x_{i}=0$, or $Z_{i}$ is inverted and $\Delta x_{i}=0$. A zoid $Z$ is minimal if all its $Z_{i}$ 's are minimal.

Given the shape $S$ of a $d$-dimensional stencil (as described in

\footnotetext{
${ }^{1}$ Frigo and Strumpen $[16,17]$ define width as the average of the two bases.
}

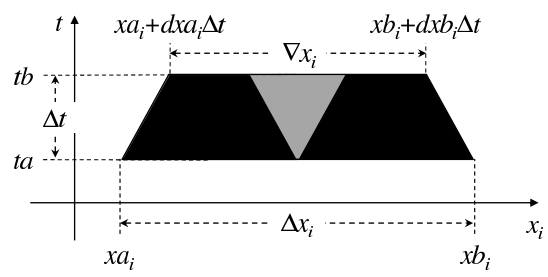

(a)

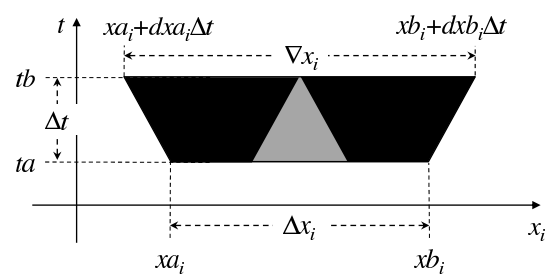

(b)

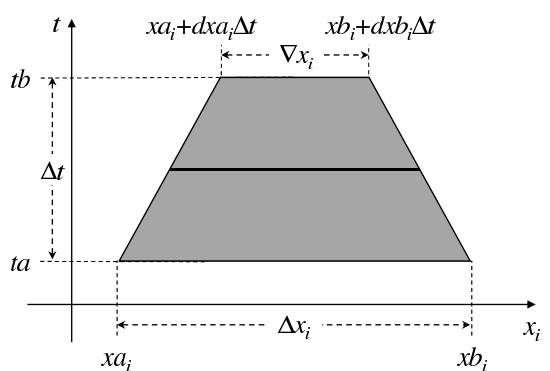

(c)

Figure 7: Cutting projection trapezoids. The spatial dimension increases to the right, and the time runs upward. (a) Trisecting an upright trapezoid using a parallel space cut produces two black trapezoids that can be processed in parallel and a gray trapezoid that must be processed after the black ones. (b) Trisecting an inverted trapezoid using a parallel space cut produces two black trapezoids that can be processed in parallel and a gray trapezoid that must be processed before the black ones. (c) A time cut produces a lower and an upper trapezoid where the lower trapezoid must be processed before the upper.

Section 2), define $t_{\text {home }}$ be the time index of the home cell. We define the slope $e^{2}$ of a cell $c=\left(t, x_{0}, x_{1}, \ldots, x_{d-1}\right) \in S$ along dimension $i \in\{0,1, \ldots, d-1\}$ as $\sigma_{i}(c)=\left|x_{i} /\left(t_{\text {home }}-t\right)\right|$, and we define the slope of the stencil along spatial dimension $i$ as $\sigma_{i}=$ $\max _{c \in S}\left\lceil\sigma_{i}(c)\right\rceil$. (Pochoir assumes for simplicity that the stencil is symmetric in each dimension.) We define the normalized width of a zoid $Z$ along dimension $i$ by $\widehat{w}_{i}=w_{i} / 2 \sigma_{i}$.

\section{Parallel space cuts}

Our trapezoidal decomposition differs from that of Strumpen and Frigo in the way we do parallel space cuts. A parallel space cut can be applied along a given spatial dimension $i$ of a well-defined zoid $z$ provided that the projection trapezoid $Z_{i}$ can be trisected into 3 well-defined subtrapezoids, as shown in Figures 7(a) and 7(b). The triangle-shaped gray subtrapezoid that lies in the middle is a minimal trapezoid. The larger base of $Z_{i}$ is split in half with each half forming the larger base of a black subtrapezoid. These three subtrapezoids of $Z_{i}$ correspond to three subzoids of $Z$. Since the two black subzoids have no interdependencies, they can be processed in parallel. As shown in Figure 7(a), for an upright projection trapezoid, the subzoids corresponding to the black trapezoids are processed first, after which the subzoid corresponding to the gray subtrapezoid can be processed. For an inverted projection trape-

\footnotetext{
${ }^{2}$ Actually, the reciprocal of slope, but we follow Frigo and Strumpen's terminology.
} 


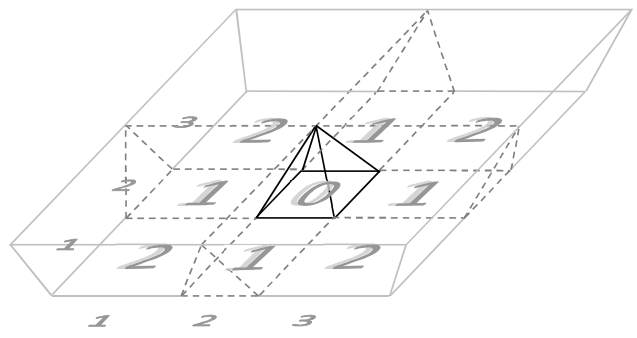

(a)

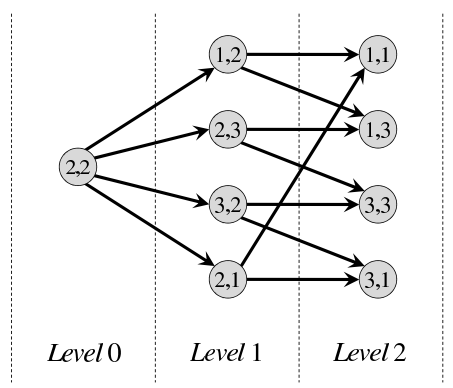

(b)

Figure 8: Dependency levels of subzoids resulting from a hyperspace cut along both spatial dimensions of a 3-zoid. (a) Labeling of coordinates of subzoids and their dependency levels. (b) The corresponding dependency graph.

zoid, as shown in Figure 7(b), the opposite is done. In either case, the 3 subzoids can be processed in parallel in the time to process 2 of them, what we shall call 2 parallel steps. The following lemma describes the general case.

LEMMA 1. All $3^{k}$ subzoids created by a hyperspace cut on $k \geq 1$ of the $d \geq k$ spatial dimensions of $a(d+1)$-zoid $Z$ can be processed in $k+1$ parallel steps.

PROOF. Assume without loss of generality that the hyperspace cut is applied to the first $k$ spatial dimensions of $z$. For each such dimension $i$, label the projection subtrapezoids in 2D space-time resulting from the parallel space cut (see Figures 7(a) and 7(b)) with the numbers 1,2 , and 3 , where the black trapezoids are labeled 1 and 3 and the gray trapezoid is labeled 2. When the hyperspace cut consisting of all $k$ parallel space cuts is applied, it creates a set $S$ of $3^{k}$ subzoids in $(k+1)$-dimensional space-time. Each subzoid can be identified by a unique $k$-tuple $\left\langle u_{0}, u_{1}, \ldots, u_{k-1}\right\rangle$, where $u_{i} \in$ $\{1,2,3\}$ for $i=0,1, \ldots, k-1$. Let $I_{i}=1$ if the projection trapezoid $z_{i}$ along the $i$ th dimension is upright and $I_{i}=0$ if $z_{i}$ is inverted. The dependency level of a zoid $\left\langle u_{0}, u_{1}, \ldots, u_{k-1}\right\rangle \in S$ is given by

$$
\operatorname{dep}\left(\left\langle u_{0}, u_{1}, \ldots, u_{k-1}\right\rangle\right)=\sum_{i=0}^{k-1}\left(\left(u_{i}+I_{i}\right) \bmod 2\right) .
$$

Observe that this equation implies exactly $k+1$ dependency levels, since each term of the summation may be either 0 or 1 . Figure 8(a) shows the dependency levels for the subzoids of a 3-zoid, both of whose projection trapezoids are inverted, generated by a hyperspace cut with $k=2$.

We claim that all zoids in $S$ with the same dependency level are independent, and thus all of $S$ can be processed in $k+1$ parallel steps. As illustrated in Figure 8(b), we can construct a directed graph $G=(S, E)$ that captures the dependency relationships among the subzoids of $S$ as follows. Given any pair of zoids $\left\langle u_{0}, u_{1}, \ldots, u_{k-1}\right\rangle,\left\langle u_{0}^{\prime}, u_{1}^{\prime}, \ldots, u_{k-1}^{\prime}\right\rangle \in S$, we include an edge $\left(\left\langle u_{0}, u_{1}, \ldots, u_{k-1}\right\rangle,\left\langle u_{0}^{\prime}, u_{1}^{\prime}, \ldots, u_{k-1}^{\prime}\right\rangle\right) \in E$, meaning that a grid point in $\left\langle u_{0}^{\prime}, u_{1}^{\prime}, \ldots, u_{k-1}^{\prime}\right\rangle$ directly depends on a grid point in $\left\langle u_{0}, u_{1}, \ldots, u_{k-1}\right\rangle$, if there exists a dimension $i \in\{0,1, \ldots, k-1\}$ such that the following conditions hold:

- $u_{j}=u_{j}^{\prime}$ for all $j \in\{0,1, \ldots, i-1, i+1, \ldots, k-1\}$,

- $\left(I_{i}+u_{i}\right) \bmod 2=0$,

- $\left(I_{i}+u_{i}^{\prime}\right) \bmod 2=1$.

Under these conditions, we have $\operatorname{dep}\left(\left\langle u_{0}^{\prime}, u_{1}^{\prime}, \ldots, u_{k-1}^{\prime}\right\rangle\right)=$ $\operatorname{dep}\left(\left\langle u_{0}, u_{1}, \ldots, u_{k-1}\right\rangle\right)+1$. Thus, along any path in $G$, the dependency levels are strictly increasing, and no two nodes with the same dependency level can lie on the same path. As a result, all zoids in $S$ with the same dependency level form an antichain and can be processed simultaneously. Thus, all zoids in $S$ can be processed in $k+1$ parallel steps with step $s \in\{0,1, \ldots, k\}$ processing all zoids having dependency level $s$.

\section{Pochoir's cache-oblivious parallel algorithm}

Given a well-defined zoid $Z$, the algorithm TRAP from Figure 2 works by recursively decomposing $Z$ into smaller well-defined zoids as follows.

Hyperspace cut. Lines 4-10 in Figure 2 apply a hyperspace cut involving all dimensions on which a parallel space cut can be applied, as shown in Figures 7(a) and 7(b). If the number $k$ of dimensions of $Z$ on which a space cut can be applied is at least 1 , as tested for in line 11 of Figure 2, then dependency levels are computed for all resulting subzoids in line 12, and then lines 13-15 recursively process them in order according to dependency level as described in the proof of Lemma 1.

Time cut. If a hyperspace cut is not applicable and $Z$ has height greater than 1 , as tested for in line 16 , then lines $17-19$ cut $Z$ in the middle of its time dimension and recursively process the lower subzoid followed by the upper subzoid, as shown in Figure 7(c).

Base case. If neither a hyperspace cut nor a time cut can be applied, lines $20-28$ processes $Z$ directly by invoking the stencilspecific kernel function. In practice, the base case is coarsened (see Section 4) by choosing a suitable threshold larger than 1 for $\Delta t$ in line 16, which cuts down on overhead due to the recursion.

\section{Analysis}

We can analyze the parallelism using a work/span analysis [7, Ch. 27]. The work $T_{1}$ of a computation is its serial running time, and the span $T_{\infty}$ is the longest path of dependencies, or equivalently, the running time on an infinite number of processors assuming no overheads for scheduling. The parallelism of a computation is the ratio $T_{1} / T_{\infty}$ of work to span.

The next lemma provides a tight bound on the span of TRAP algorithm on a minimal zoid.

LEMMA 2. Consider a minimal $(d+1)$-zoid $Z$ with height $h$ and normalized widths $\widehat{w}_{i}=h$ for $i \in\{0,1, \ldots, d-1\}$. Then the span of TRAP when processing $Z$ is $\Theta\left(d h^{\lg (d+2)}\right)$.

Proof. For simplicity we assume that a call to the kernel function costs $O(1)$, as in [17]. As TRAP processes $Z$, some of the subzoids generated recursively have normalized widths equal to their heights and some have twice that amount. Let us denote by $T_{\infty}(h, k, d-k)$ the span of TRAP processing a $(d+1)$-zoid with height $h$ where $k \geq 0$ of the $d$ spatial dimensions have normalized width $2 h$ and $d-k$ spatial dimensions have normalized width $h$. Using Lemma 1, the span of TRAP processing a zoid $Z$ when it undergoes a hyperspace cut can be described by the recurrence

$$
\begin{aligned}
T_{\infty}(h, k, d-k) & =(k+1) T_{\infty}(h, 0, d)+\Theta\left(\sum_{i=0}^{k} \lg \left(3^{k}\right)\right) \\
& =(k+1) T_{\infty}(h, 0, d)+\Theta\left(k^{2}\right),
\end{aligned}
$$


where $T(1,0, d)=\Theta(1)$ is the base case. The summation in this derivation represents the span due to spawning. A parallel for with $r$ iterations adds $\Theta(\lg r)$ to the span, and since the number of zoids at all levels is $3^{k}$, this value upper-bounds the number of iterations at any given level. Moreover, the lower bound on the number of zoids on a given level is at least the average $3^{k} /(k+1)$, whose logarithm is asymptotically the same as $\lg \left(3^{k}\right)$, and hence the bound is asymptotically tight.

A time cut can be applied when the zoid $Z$ is minimal. Assume that $k \geq 0$ projection trapezoids $Z_{i}$ 's are upright and the rest are inverted. Then for each upright projection trapezoid $Z_{i}$, the normalized width of the lower zoid generated by the hyperspace cut is $\widehat{w}_{i}=h$, the same as for $Z$, and for each inverted projection trapezoid $z_{i}$, the lower zoid has normalized width $\widehat{w}_{i}-h / 2=h / 2$. Similarly, for each upright projection trapezoid $z_{i}$, the normalized width of the upper zoid is $\widehat{w}_{i}-h / 2=h / 2$, and for each inverted projection trapezoid $Z_{i}$, the upper zoid has normalized width $\widehat{w}_{i}$. Thus, the recurrence for the span of TRAP when a minimal $z$ undergoes a time cut can be written as follows:

$$
T_{\infty}(h, 0, d)=T_{\infty}(h / 2, k, d-k)+T_{\infty}(h / 2, d-k, k)+\Theta(1) .
$$

Applying hyperspace cuts to the subzoids on the right-hand side of this recurrence yields

$$
\begin{aligned}
T_{\infty}(h, 0, d) & =(d+2) T_{\infty}(h / 2,0, d)+\Theta\left(k^{2}\right)+\Theta\left((d-k)^{2}\right) \\
& =(d+2) T_{\infty}(h / 2,0, d)+\Theta\left(d^{2}\right) \\
& =\Theta\left(d^{2}(d+2)^{\lg h-1}\right)+\Theta\left((d+2)^{\lg h}\right) \\
& =\Theta\left(d h^{\lg (d+2)}\right) .
\end{aligned}
$$

THEOREM 3. Consider $a(d+1)$-dimensional grid $Z$ with $\widehat{w}_{i}=$ $w$ for $i \in\{0,1, \ldots, d-1\}$ and height $h=2^{r} w$. Then the parallelism of TRAP when processing Z using a stencil with constant slopes is $\Theta\left(w^{d-\lg (d+2)+1} / d^{2}\right)$.

Proof. Assume without loss of generality that the stencil is periodic. (As will be discussed in Section 4, Pochoir implements TRAP so that the control structure for nonperiodic stencils is the same as that for periodic.) The algorithm first applies a series of $r$ time cuts, dividing the original time dimension into $h / w=2^{r}$ subgrids with $\widehat{w}_{i}=w$ with height $w$. These grids are processed serially. The next action of TRAP applies a hyperspace cut to all $d$ spatial dimensions of $Z$, dividing the grid into $d+1$ minimal zoids which are then processed serially. Applying Lemma 2 yields a span of

$$
\begin{aligned}
T_{\infty} & =(h / w)(d+1) \cdot \Theta\left(d w^{\lg (d+2)}\right) \\
& =\Theta\left(\left(d^{2} h\right) w^{\lg (d+2)-1}\right) .
\end{aligned}
$$

The work is the volume of $Z$, which is $T_{1}=\Theta\left(h w^{d}\right)$, since the stencil has constant slopes. Thus, the parallelism is

$$
T_{1} / T_{\infty}=\Theta\left(w^{d-\lg (d+2)+1} / d^{2}\right) .
$$

We can compare TRAP with a version of Frigo and Strumpen's parallel stencil algorithm [17] we call STRAP, which performs the space cuts serially as in Figures 7(a) and 7(b). Each space cut results in one synchronization point, and hence a sequence of $k$ space cuts applied by STRAP introduces $2^{k}$ parallel steps compared to the $k+1$ parallel steps generated by TRAP (see Lemma 1). Thus, each space cut virtually doubles STRAP's span. Figure 8(a) shows a simple example where STRAP produces $2^{2}-1=3$ synchronization points while TRAP introduces only 2 . The next lemma and theorem analyze STRAP, mimicking Lemma 2 and Theorem 3. Their proofs are omitted.
LEMMA 4. Consider a minimal $(d+1)$-zoid $Z$ with height $h$ and normalized widths $\widehat{w}_{i}=h$ for $i \in\{0,1, \ldots, d-1\}$. Then the span of STRAP when processing $Z$ is $\Theta\left(h^{\lg \left(2^{d}+1\right)}\right)$.

THEOREM 5. Consider $a(d+1)$-dimensional grid $Z$ with $\widehat{w}_{i}=$ $w$ for $i \in\{0,1, \ldots, d-1\}$ and height $h=2^{r} w$. Then the parallelism of STRAP when processing $Z$ using a stencil with constant slopes is $\Theta\left(w^{d-\lg \left(2^{d}+1\right)+1} / 2^{d}\right)$.

\section{Discussion}

As can be seen from Theorems 3 and 5, both TRAP and STRAP have the same asymptotic parallelism $\Theta\left(w^{2-\lg 3}\right)$ for $d=1$, but for $d=2$, TRAP has $\Theta\left(w^{2}\right)$ while STRAP has $\Theta\left(w^{3-\lg 5}\right)$, and the difference grows with the number of dimensions.

The cache complexities of TRAP and STRAP are the same, which follows from the observation that both algorithms apply exactly the same time cuts in exactly the same order, and immediately before each time cut, both are in exactly the same state in terms of the spatial cuts applied. Thus, they arrive at exactly the same configuration - number, shape, and size — of subzoids before each time cut.

Frigo and Strumpen's parallel stencil algorithm is actually slightly different from STRAP. For any fixed integer $r>1$, a space cut in their algorithm produces $r$ black zoids and between $r-1$ and $r+1$ gray zoids. STRAP is a special case of that algorithm with $r=2$ for upright projection trapezoids and $r=1$ for inverted projection trapezoids. For larger values of $r$, Frigo and Strumpen's algorithm achieves more parallelism but the cache efficiency drops. It is straightforward to extend TRAP to perform $r$ multiple cuts along each dimension to match the cache complexity of Frigo and Strumpen's algorithm while providing asymptotically more parallelism.

\section{Empirical results}

Figure 9 shows the results of using the Cilkview scalability analyzer [20] to compare the parallelism of TRAP and STRAP on two typical benchmarks. We measured the two algorithms with uncoarsened base cases. As can be seen from the figure, TRAP's asymptotic advantage in parallelism is borne out in practice for these benchmarks.

We used the Linux perf tool [29] to verify that TRAP does not suffer any loss in cache efficiency compared to the STRAP algorithm. Figure 10 also plots the cache-miss ratio of the straightforward parallel loop algorithm, showing that it exhibits poorer cache performance than the two cache-oblivious algorithms.

\section{COMPILER OPTIMIZATIONS}

The Pochoir compiler transforms code written in the Pochoir specification language into optimized $\mathrm{C}++$ code that employs the Intel Cilk multithreading extensions [23]. The Pochoir compiler is written in Haskell [37], and it performs numerous optimizations, the most important of which are code cloning, loop-index calculations, unifying periodic and nonperiodic boundary conditions, and coarsening the base case of recursion. This section describes how the Pochoir compiler implements these optimizations.

Before a programmer compiles a stencil code with the Pochoir compiler, he or she is expected to perform Phase 1 of Pochoir's twophase methodology which requires that it be compiled using the Pochoir template library and debugged. This $\mathrm{C}++$ template library is employed by both Phases 1 and 2 and includes both loop-based and trapezoidal algorithms. Differences between stencils, such as dimensionality or data structure, are incorporated into these generic algorithms at compile-time via $\mathrm{C}++$ template metaprogramming. 


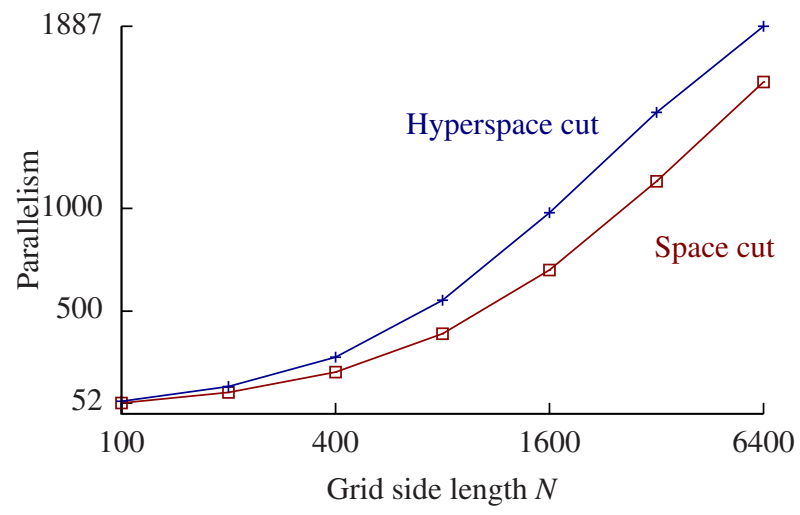

(a)

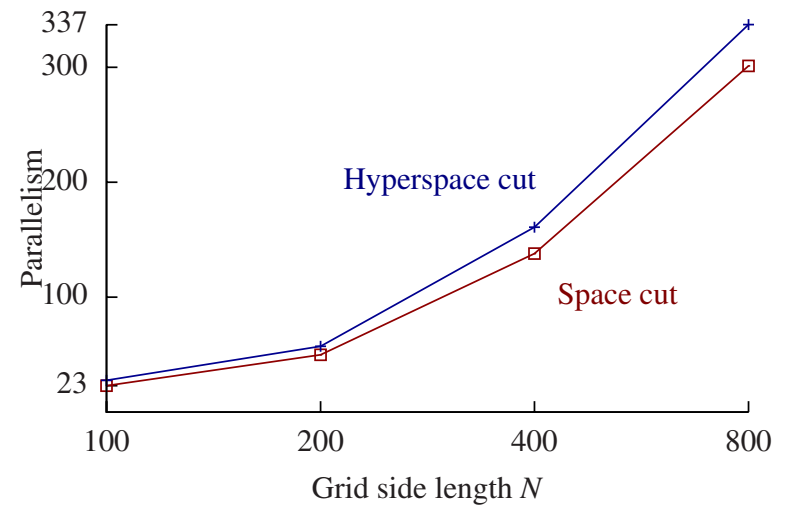

(b)

Figure 9: Parallelism comparison on two benchmarks between TRAP, which employs hyperspace cuts, and STRAP, which uses serial space cuts. Measurements are of code without base-case coarsening. (a) 2D nonperiodic heat equation. Space-time size is $1000 N^{2}$. (b) 3D nonperiodic wave equation. Space-time size is $1000 N^{3}$.

\section{Handling boundary conditions by code cloning}

The handling of boundary conditions can easily dominate the runtime of a stencil computation. For example, we coded the $2 \mathrm{D}$ heat equation on a periodic torus using Pochoir, and we compared it to a comparable code that simply employs a modulo operation on every array index. For a $5000^{2}$ spatial grid over 5000 time steps, the runtime of the modular-indexing implementation degraded by a factor of 2.3 .

For nonperiodic stencil computations, where a value must be provided on the boundary, performance can degrade even more if a test is made at every point to determine whether the index falls off the grid. Stencil implementers often handle constant nonperiodic boundary conditions with the simple trick of introducing ghost cells [8] that form a halo around the periphery of the grid. Ghost cells are read but never written. The stencil computation can apply the kernel function to the grid points on the real grid, and accesses that "fall off" the edge into the halo obtain their values from the ghost cells without any need to check boundary conditions.

In practice, however, nonperiodic boundary conditions can be more complicated than simple constants, and we wanted to allow Pochoir users flexibility in the kinds of boundary conditions they could specify. For example, Dirichlet boundary conditions may specify boundary values that change with time, and Neumann boundary conditions may specify the value the derivative should

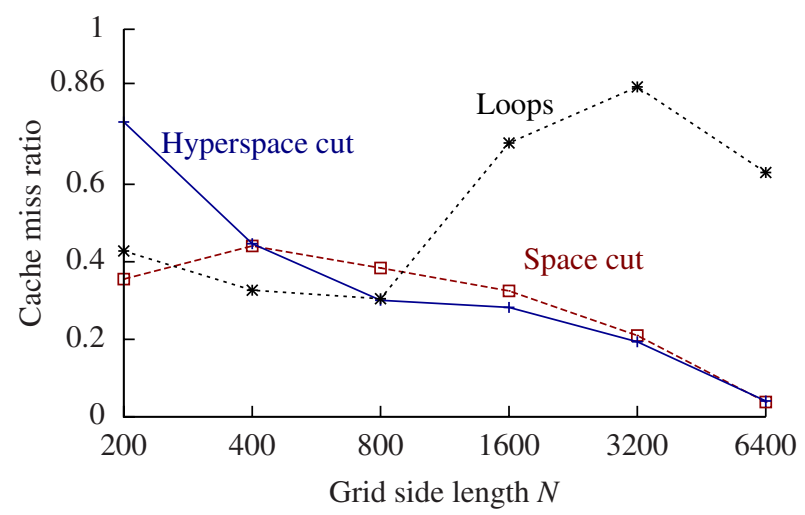

(a)

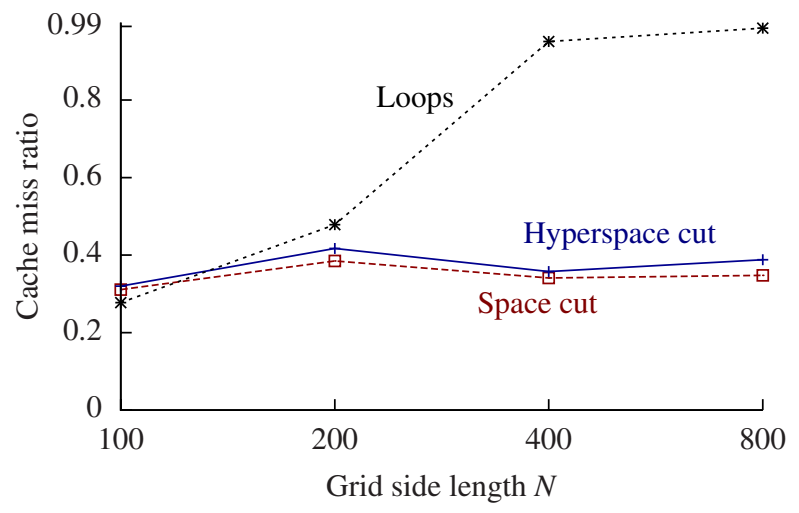

(b)

Figure 10: Cache-miss ratios for two benchmarks using TRAP, STRAP, and a parallel-loop algorithm. The cache-miss ratio is the ratio of the cache misses to the number of memory references. Measurements are of code without base-case coarsening. (a) $2 \mathrm{D}$ nonperiodic heat equation. Spacetime is $1000 N^{2}$. (b) 3D nonperiodic wave equation. Space-time is $1000 N^{3}$.

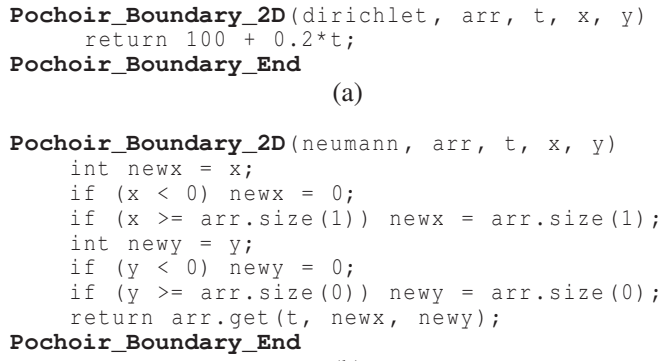

(a)

(b)

Figure 11: Pochoir code for specifying nonperiodic boundary conditions. (a) A Dirichlet condition with constrained boundary value (set equal to a function of $t$ ). (b) A Neumann condition with constrained derivative at the boundary (set equal to 0 ).

take on the boundary [14]. Figure 11(a) shows a Pochoir specification of a Dirichlet boundary condition, and Figure 11(b) shows the Pochoir specification of a Neumann boundary condition.

To handle boundaries efficiently, the Pochoir compiler generates two code clones of the kernel function: a slower boundary clone and a faster interior clone. The boundary clone is used for boundary zoids: those that contain at least one point whose computation requires an off-grid access. The interior clone is used for interior zoids: those all of whose points can be updated without indexing 
kernel function is invoked. When a zoid wraps around the grid in a given dimension $i$, meaning that $x a_{i}>x b_{i}$, we represent the lowerand upper-bound coordinates of the zoid in dimension $i$ by virtual coordinates $\left(x a_{i}, N_{i}+x b_{i}\right)$, where $N_{i}$ is the size of the periodic grid in dimension $i$. In the base of the recursion of the boundary clone, Pochoir calls the kernel function and supplies it with the true coordinates of the grid point being updated by performing a modulo computation on each coordinate. Within the kernel function, accesses to the Pochoir arrays now call the boundary function, which provides the correct value for grid points that are outside the true grid. Of course, no such checking is required for interior zoids, which are always represented by true coordinates.

\section{Coarsening of base cases}

Previous work [9, 26, 27, 34] has found that although trapezoidal decomposition dramatically reduces cache-miss rates, overall performance can suffer from function-call overhead unless the base case of the recursion is coarsened. For example, proper coarsening of the base case of the 2D heat-equation stencil (running for 5000 time steps on a $5000 \times 5000$ toroidal grid) improves the performance by a factor of 36 over running the recursion down to a single grid point.

Since choosing the optimal size of the base case can be difficult, we integrated the ISAT autotuner [22] into Pochoir. Despite the advantage of finding the optimal coarsening factor on any specific platform, this autotuning process can take hours to find the optimal value, which may be unacceptable for some users.

In practice, Pochoir employs some heuristics to choose a reasonable coarsening. One principle is that to maximize data reuse, we want to make the spatial dimensions all about the same size. Another principle is that to exploit hardware prefetching, we want to avoid cutting the unit-stride spatial dimension and avoid oddshaped base cases. For example, for 2D problems, a square-shaped computing domain often offers the best performance. We have found that for $3 \mathrm{D}$ problems, the effect of hardware prefetching can often be more important than cache efficiency for reasonably sized base cases. Consequently, for 3 or more dimensions, Pochoir adopts the strategy of never cutting the unit-stride spatial dimension, and it cuts the rest of the spatial dimensions into small hypercubes to ensure that the entire base case stays in cache. Given all that potential complexity, the compiler's heuristic is actually fairly simple. For 2D problems, Pochoir stops the recursion at $100 \times 100$ space chunks with 5 time steps. For $3 \mathrm{D}$ problems, the recursion stops at $1000 \times 3 \times 3$ with 3 time steps.

\section{RELATED WORK}

Attempts to compile stencils into highly optimized code are not new. This section briefly reviews the history of stencil compilers and discusses some of the more recent innovative strategies for optimizing stencil codes.

Special-purpose stencil compilers for distributed-memory machines first came into existence at least two decades ago [3, 4, 39]. The goal of these researchers was generally to reduce interprocessor data transfer and improve the performance of loop-based stencil computations through loop-level optimizations. The compilers expected the stencils to be expressed in some normalized form.

More recently, Krishnamoorthy et al. [28] have considered automatic parallelization of loop-based stencil codes through loop tiling, focusing on load-balancing the execution of the tiles. Kamil et al. [25] have explored automatic parallelization and tuning of stencil computations for chip multiprocessors. The stencils are specified using a domain-specific language which is a subset of Fortran 95. An abstract syntax tree is built from the stencil specified in the input language, from which multiple formats of output can be generated, including Fortran, C, and CUDA. The parallelization is based on blocked loops.

We have discussed Frigo and Strumpen's seminal trapezoidaldecomposition algorithms [16,17] at length, since they form the foundation of the Pochoir algorithm. Nitsure [34] has studied how to use Frigo and Strumpen's parallel algorithm to implement 2D and 3D lattice Boltzmann methods. In addition to several other optimizations, Nitsure employs two code clones for the kernel to reduce the overhead of boundary checking, which Pochoir does as well. Nitsure's stencil code is parallelized with OpenMP [35], and data dependencies among subdomains are maintained by locking.

Cache-aware techniques have been used extensively to improve the stencil performance. Datta et al. [9] and Kamil et al. [26, 27] have applied both algorithmic and coding optimizations to loopbased stencil computations. Their algorithmic optimizations include an explicitly blocked time-skewing algorithm which overlaps subregions to improve parallelism at the cost of redundant memory storage and computation. Their coding optimizations include processor-affinity binding, kernel inlining, an explicit user stack, early cutoff, indirection instead of modulo, and autotuning.

Researchers at the University of Southern California [11, 12,36] have performed extensive studies on how to improve the performance of high-order stencil computations though parallelization and optimization. Their techniques, which apply variously to multicore and cluster machines, include intranode, internode, and dataparallel optimizations, such as cache blocking, register blocking, manual SIMD-izing, and software prefetching.

\section{CONCLUDING REMARKS}

It is remarkable how complex a simple computation can be when performance is at stake. Parallelism and caching make stencil computations interesting. As discussed in Section 5, many researchers have investigated how various other features of modern machines - such as prefetching units, graphical processing units, and clustering — can be exploited to provide even more performance. We see many ways to improve Pochoir by taking advantage of these machine capabilities.

In addition, we see ample opportunity to enhance the linguistic features of the Pochoir specification language to provide more generality and flexibility to the user. For example, we are considering how to allow the user to specify irregularly shaped domains. As long as the boundary of a region, however irregular, is small compared to the region's interior, special-case code to handle the boundary should not adversely impact the overall performance. Even more challenging is coping with boundaries that change with time. We believe that such capabilities will dramatically speed up the PSA, RNA, and LCS benchmarks which operate on diamondshaped space-time domains.

Pochoir's two-phase compilation strategy introduces a new method for building domain-specific languages embedded in $\mathrm{C}++$. Historically, the complexity of parsing and type-checking $\mathrm{C}++$ has impeded such separately compiled domain-specific languages. C++'s template programming does provide a good measure of expressiveness for describing special-purpose computations, but it provides no ability to perform the domain-specific optimizations such as those that Pochoir employs. Pochoir's compilation strategy offers a new way to build optimizing compilers for domain-specific languages embedded in $\mathrm{C}++$ where the compiler can parse and "understand" only as much of the programmer's C++ code as it is able, confident that code it does not understand is nevertheless correct.

The Pochoir compiler can be downloaded from http:// supertech.csail.mit.edu/pochoir. 


\section{ACKNOWLEDGMENTS}

Thanks to Matteo Frigo of Axis Semiconductor and Volker Strumpen of the University of Linz, Austria, for providing us with their code for trapezoidal decomposition of the 2D heat equation which served as a model and inspiration for Pochoir. Thanks to Kaushik Datta of Reservoir Labs and Sam Williams of Lawrence Berkeley National Laboratory for providing us with the Berkeley autotuner code and help with running it. Thanks to Geoff Lowney of Intel for his support and critical appraisal of the system and to Robert Geva of Intel for an enormously helpful discussion that led to a great simplification of the Pochoir specification language. Many thanks to the Intel Cilk team for support during the development of Pochoir, and especially Will Leiserson for his responsiveness as the SPAA submission deadline approached. Thanks to Will Hasenplaugh of Intel and to members of the MIT Supertech Research Group for helpful discussions.

\section{REFERENCES}

[1] T. Akutsu. Dynamic programming algorithms for RNA secondary structure prediction with pseudoknots. Discrete Applied Mathematics, 104:45-62, 2000.

[2] R. Bleck, C. Rooth, D. Hu, and L. T. Smith. Salinity-driven thermocline transients in a wind- and thermohaline-forced isopycnic coordinate model of the North Atlantic. Journal of Physical Oceanography, 22(12):1486-1505, 1992.

[3] R. G. Brickner, W. George, S. L. Johnsson, and A. Ruttenberg. A stencil compiler for the Connection Machine models CM-2/200. In Workshop on Compilers for Parallel Computers, 1993.

[4] M. Bromley, S. Heller, T. McNerney, and G. L. Steele Jr. Fortran at ten Gigaflops: The Connection Machine convolution compiler. In PLDI, pages 145-156, Toronto, Ontario, Canada, June 26-28 1991.

[5] C++ Standards Committee. Working draft, standard for programming language C++. available from http://www.open-std.org/jtc1/ sc22/wg21/docs/papers/2011/n3242.pdf, 2011. ISO/IEC Document Number N3242=11-0012.

[6] R. A. Chowdhury, H.-S. Le, and V. Ramachandran. Cache-oblivious dynamic programming for bioinformatics. TCBB, 7(3):495-510, July-Sept. 2010.

[7] T. H. Cormen, C. E. Leiserson, R. L. Rivest, and C. Stein. Introduction to Algorithms. The MIT Press, third edition, 2009.

[8] K. Datta. Auto-tuning Stencil Codes for Cache-Based Multicore Platforms. PhD thesis, EECS Department, University of California, Berkeley, Dec 2009.

[9] K. Datta, M. Murphy, V. Volkov, S. Williams, J. Carter, L. Oliker, D. Patterson, J. Shalf, and K. Yelick. Stencil computation optimization and auto-tuning on state-of-the-art multicore architectures. In $S C$, pages 4:1-4:12, Austin, TX, Nov. 15-18 2008.

[10] A. van Deursen, P. Klint, and J. Visser. Domain-specific languages: An annotated bibliography. SIGPLAN Not., 35(6):26-36, June 2000.

[11] H. Dursun, K.-i. Nomura, L. Peng, R. Seymour, W. Wang, R. K. Kalia, A. Nakano, and P. Vashishta. A multilevel parallelization framework for high-order stencil computations. In Euro-Par, pages 642-653, Delft, The Netherlands, Aug. 25-28 2009.

[12] H. Dursun, K.-i. Nomura, W. Wang, M. Kunaseth, L. Peng, R. Seymour, R. K. Kalia, A. Nakano, and P. Vashishta. In-core optimization of high-order stencil computations. In PDPTA, pages 533-538, Las Vegas, NV, July13-16 2009.

[13] J. F. Epperson. An Introduction to Numerical Methods and Analysis. Wiley-Interscience, 2007.

[14] H. Feshbach and P. Morse. Methods of Theoretical Physics. Feshbach Publishing, 1981.

[15] M. Frigo, C. E. Leiserson, H. Prokop, and S. Ramachandran. Cache-oblivious algorithms. In FOCS, pages 285-297, New York, NY, Oct. 17-19 1999.

[16] M. Frigo and V. Strumpen. Cache oblivious stencil computations. In ICS, pages 361-366, Cambridge, MA, June 20-22 2005.
[17] M. Frigo and V. Strumpen. The cache complexity of multithreaded cache oblivious algorithms. Theory of Computing Systems, 45(2):203-233, 2009.

[18] M. Gardner. Mathematical Games. Scientific American, 223(4):120-123, 1970 .

[19] O. Gotoh. An improved algorithm for matching biological sequences. Journal of Molecular Biology, 162:705-708, 1982.

[20] Y. He, C. E. Leiserson, and W. M. Leiserson. The Cilkview scalability analyzer. In SPAA, pages $145-156$, Santorini, Greece, June 13-15 2010.

[21] P. Hudak. Building domain-specific embedded languages. ACM Computing Surveys, 28(4), December 1996.

[22] Intel software autotuning tool. http: //software.intel.com/enus/articles/intel-software-autotuning-tool/, 2010.

[23] Intel Corporation. Intel Cilk Plus Language Specification, 2010. Document Number: 324396-001US. Available from http://software.intel.com/sites/products/cilk-plus/ cilk_plus_language_specification.pdf.

[24] C. John. Options, Futures, and Other Derivatives. Prentice Hall, 2006.

[25] S. Kamil, C. Chan, L. Oliker, J. Shalf, and S. Williams. An auto-tuning framework for parallel multicore stencil computations. In IPDPS, pages 1-12, 2010.

[26] S. Kamil, K. Datta, S. Williams, L. Oliker, J. Shalf, and K. Yelick. Implicit and explicit optimizations for stencil computations. In MSPC, pages 51-60, San Jose, CA, 2006.

[27] S. Kamil, P. Husbands, L. Oliker, J. Shalf, and K. Yelick. Impact of modern memory subsystems on cache optimizations for stencil computations. In MSP, pages 36-43, Chicago, IL, June 122005.

[28] S. Krishnamoorthy, M. Baskaran, U. Bondhugula, J. Ramanujam, A. Rountev, and P. Sadayappan. Effective automatic parallelization of stencil computations. In PLDI, San Diego, CA, June 10-13 2007.

[29] https://perf.wiki.kernel.org/index.php/Main_Page.

[30] R. Mei, W. Shyy, D. Yu, and L. Luo. Lattice Boltzmann method for 3-D flows with curved boundary. J. of Comput. Phys, 161(2):680-699, 2000.

[31] M. Mernik, J. Heering, and A. M. Sloane. When and how to develop domain-specific languages. ACM Computing Surveys, 37:316-344, December 2005.

[32] P. Micikevicius. 3D finite difference computation on GPUs using CUDA. In GPPGPU, pages 79-84, Washington, DC, Mar. 82009.

[33] A. Nakano, R. Kalia, and P. Vashishta. Multiresolution molecular dynamics algorithm for realistic materials modeling on parallel computers. Computer Physics Communications, 83(2-3):197-214, 1994.

[34] A. Nitsure. Implementation and optimization of a cache oblivious lattice Boltzmann algorithm. Master's thesis, Institut für Informatic, Friedrich-Alexander-Universität Erlangen-Nürnberg, July 2006.

[35] OpenMP application program interface, version 2.5. OpenMP specification, May 2005.

[36] L. Peng, R. Seymour, K.-i. Nomura, R. K. Kalia, A. Nakano, P. Vashishta, A. Loddoch, M. Netzband, W. R. Volz, and C. C. Wong. High-order stencil computations on multicore clusters. In IPDPS, pages 1-11, Rome, Italy, May 23-29 2009.

[37] S. Peyton Jones. Haskell 98 Language and Libraries: The Revised Report. Cambridge University Press, 1998.

[38] H. Prokop. Cache-oblivious algorithms. Master's thesis, Department of Electrical Engineering and Computer Science, Massachusetts Institute of Technology, June 1999.

[39] G. Roth, J. Mellor-Crummey, K. Kennedy, and R. G. Brickner. Compiling stencils in High Performance Fortran. In $S C$, pages 1-20, San Jose, CA, Nov. 16-20 1997. ACM.

[40] A. Taflove and S. Hagness. Computational Electrodynamics: The Finite-Difference Time-Domain Method. Artech House, Norwood, MA, 2000

[41] S. Williams, J. Carter, L. Oliker, J. Shalf, and K. Yelick. Lattice Boltzmann simulation optimization on leading multicore platforms. In IPDPS, pages 1-14, Miami, FL, Apr. 2008. 